\title{
Corporate involvement in public health policy is being obscured
}

The competing interests section of this Editorial lists an incorrect grant number (BMJ 2013;346:f3429, doi:10.1136/bmj.f3429). The authors acknowledged that their work derived from research supported by the National Cancer Institute of the US National
Institutes of Health, but the grant number should have read R01 CA160695-01 (not “2 R01 CA091021-05,” as was published).

Cite this as: BMJ 2013;346:f3766

๑ BMJ Publishing Group Ltd 2013 